\title{
Simulation and Calibration of Hydro-Debris 2D Model (HD2DM) to Predict the Particle Segregation Processes
}

\section{in Debris Flow}

\author{
Yosuke Yamashiki ${ }^{1,2}$, Mohd Remy Rozainy M. A. Z. ${ }^{3}$, Taku Matsumoto ${ }^{4}$, Tamotsu Takahashi ${ }^{5}$ and Kaoru \\ Takara $^{1}$ \\ 1. Department of Urban and Environmental Engineering, Kyoto University, Kyoto 615-8530, Japan \\ 2. Science and Technology Research Partnership for Sustainable Development, Tokyo 102-0076, Japan \\ 3. School of Civil Engineering, Universiti Sains Malaysia, Penang 14300, Malaysia \\ 4. Sumitomo Corporation, Tokyo 104-8610, Japan \\ 5. Association for Disaster Prevention Research, Kyoto 606-8226, Japan
}

\begin{abstract}
The principal aim of a vertical two-dimensional numerical model development is for estimating the particle tracing and mechanism of $10 \mathrm{~mm}$ and $2.5 \mathrm{~mm}$ debris. The particle tracing movement can be visually analyzed by using a high speed video camera (HSVC). A numerical model was developed using the Marker and Cell Method, which involves a Subgrid-Scale (SGS) model and the Particle Source in Cell (PSI-Cell) Method. The transportation processes of debris and air bubble were simulated in lagrangian form by introducing air bubbles and debris markers. Air bubble movement characteristics were simulated by this numerical model. Bigger particles flow at the upper part, while smaller particles attach near to the bottom. This phenomenon is similar to what we observed in the experimental studies. As a conclusion, the calibration processes for velocity was successful. The value of virtual mass $\left(C_{M}\right)$ was found to be one of the most important criteria that should be considered in the calibration process, as this parameter dominates fundamental characteristics of sediment particle movement in the lagrangian numerical scheme. The best fitted $\mathrm{C}_{\mathrm{M}}$ in this study was 0.35 . The mean average velocity value ranging from $1.2 \%$ to $22.61 \%$ is obtained from the velocity results of numerical studies compared to the experimental studies.
\end{abstract}

Key words: Debris flow, high speed video camera (hsvc), particle routing segregation, experimental debris flow study.

\section{Introduction}

Debris flows are characterized by multiple surges which take place intermittently and subsequently flows do not follow the course of previous flows. A debris flow therefore takes another course keeping off the lobes formed by previous flows. That is why the course of a debris flow always swings and a fan like topography is formed. The front of a debris flow makes a stop when it reaches a gradient of less than 10 degrees leaving a scale-like land form called debris lobes. Subsequent flows which contain less sediment

Corresponding author: Mohd Remy Rozainy M. A. Z., $\mathrm{PhD}$, senior lecturer, research field: water resources engineering. E-mail: ceremy@eng.usm.my. continue running incising old deposits. The resulting destruction of debris flow characterized by, first, massive impact force of the front and second, the burying capacity of valuable land, assets and infrastructures. In general large boulders running at the front carry enormous amounts of energy. This makes debris quite hazardous in terms of destructive power and the size of disaster area [1]. Large boulders, run at the front of debris flows looking like an overwhelming wall of water thus nothing but a massive concrete structure can stop them [2]. An estimation of the potential impact force of bolders from a debris flow is important for the design of structural mitigation elements. The estimation of impact forces of bolders 
are most sensitive to the inputs of particle size and particle velocity [3-5].

Modeling debris flows has gained increasing interest in recent years, due to the potential damage that these flows can generate, particularly in urbanized alluvial fans. However, hydrodynamic modeling of debris flow surges is much more challenging than that of water flows, mainly due to the multi-phase character of the flow, which includes not only water, but also a wide range of interacting solid particles, that goes from very fine sediments such as silt and clay, to large particles such as boulders; timber and other debris.

Several sets of flume experiments were examined by numerical tools to verify the appropriateness of numerical model and the validity of numerical procedures. To understand the general behavior of particle segregation in debris flow, a physical model experiment for debris flow with two distinct diameters were conducted. Furthermore, the high-speed video camera (HSVC) results tracing each particle movement are compared with the solutions of Hydro Debris 2D Model (HD2DM), a Lagrangian sediment particle tracing numerical.

The main aim of this study is to understand the characteristics movements of stony debris flow and develop a most reliable numerical model to predict the debris flow disasters routing through laboratory experiments and numerical simulation models. Therefore, this study focuses on capturing the process of debris flow particle routing segregation at a $25^{\circ}$ slope angle, particle routing segregation processes at the downstream of the rectangular channel and development of debris flow numerical computational model by Lagrangian concept. The calibration of the HD2DM based on the experimental study of hydraulic physical model had been done in this study.

\section{Laboratory Experiments}

In order to understand the characteristics of debris flow routing mechanism and the deposition behavior, it is necessary to set up a debris flow experimental physical model. The model consists of three main parts which are the rectangular flume, deposition board and water intake tank. The debris was placed $3.5 \mathrm{~m}$ from the bottom of the rectangular flume. The detail of debris load in the rectangular flume is shown in Fig. 1. The debris was well mixed up between small and big materials. At least three time of experiment had been made to understand the stony particle distribution and movements. After a flume and board were set to the prescribed slopes, a constant discharge was supplied from the upstream end of the channel through an electromagnetic valve (gate). A constant discharge (3.0 $\mathrm{L} / \mathrm{s}$ ) is supplied within $7 \mathrm{~s}$.

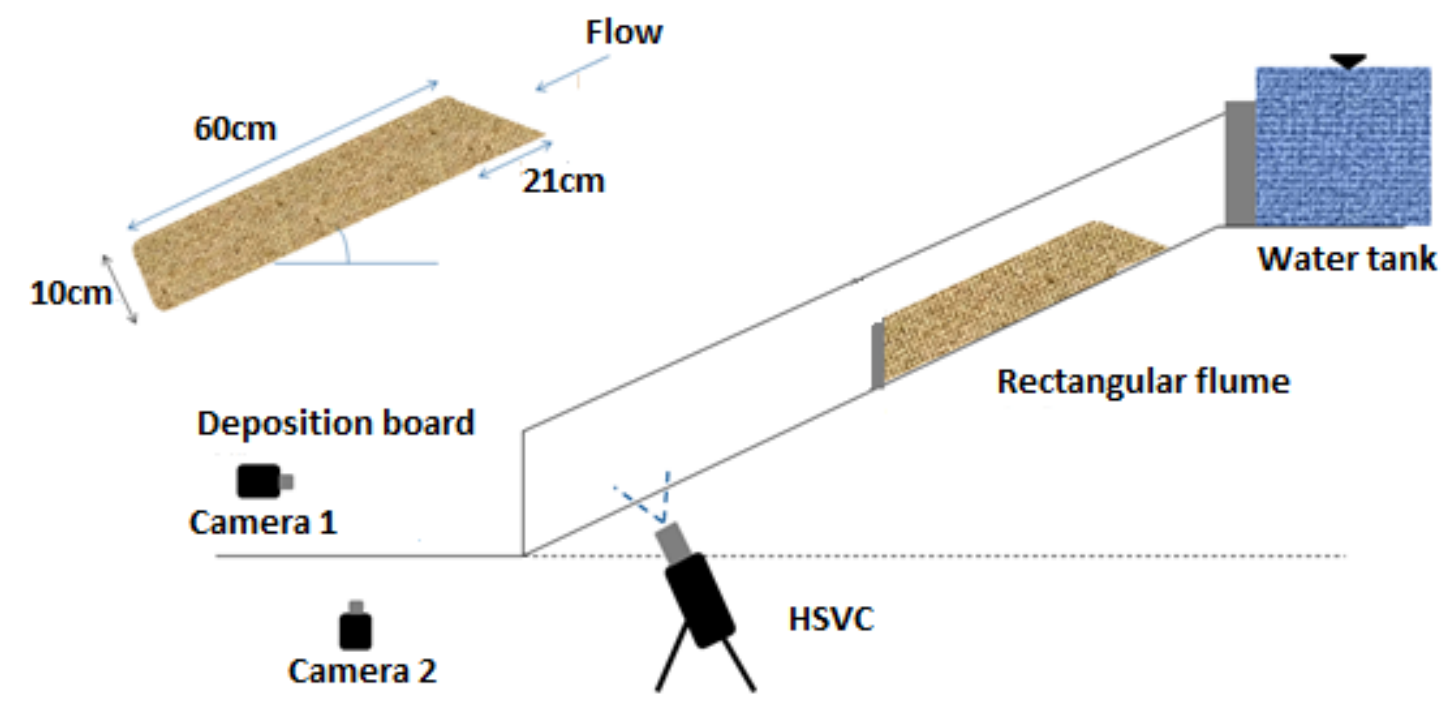

Fig. 1 The schematic diagram of experimental procedure. 
During water supply, HSVC will record the image of particle routing. The HSVC have been placed at one location which is near the downstream the rectangular flume. The HSVC can capture a video footage during short intervals time (0-9 s). Moreover, two video cameras were set at different locations to record continuous and simultaneous process of debris flow deposition process. Video recording of the experiments are performed to analyzed debris flow characteristics and captured the formation of debris flow deposition process. Four groups of images had been classified such as initial (no flow), starting with flow, intermediate (2 s) and last (4 s). The images of particle tracing were captured between $0.015 \mathrm{~s}$. The distance of particle movements were identified. By knowing the distance of each particle distribution, the velocity of each particle can be calculated.

\section{Numerical Model of Hydro-debris 2D Model}

The Marker and Cell method was modified, combining SGS model and PSI-CELL method [6, 7]. A lagrangian solution for the air bubble transport was determined by surrounding water pressure with assumption of initially uniform air bubbles diameters. The mechanism of picking up and transport process of bed material in macro turbulence zone was simulated using the lagrangian model based on Tchen's equation [8] and Gotoh's [6], assuming that bed materials is uniform. The computer code was written in C language, using reference "103 Y0/VIFMAC" of the computer library of Kyoto University, created by Takemoto [9] and developed by Sakai et al. [10]. The systems of governing equations are the grid-filtered time-dependent three-dimensional compressible (with low Mach number) mixed flow Navier-Stokes, liquid phase continuity equations. SGS (Sub Grid Scale) model for only liquid phase is introduced. The effect of Lagrangian sediment particle into liquid phase is being considered using PSI-CELL (particle source in cell) method [6, 7].

\subsection{Eulerian Model}

Several researchers have estimates of sediment transport by focusing on the existence of turbulence. The largest contribution was made by Rijn and Leo [11, 12]. The theory of sediment transport for laminar flow was developed by Van Rijn using relatively simple calculation. Yet, it shows great feasibility in the turbulence model ( $\kappa-\varepsilon$ model).

The two characteristic parameters had been introduced

(1) Particle parameter

$$
D_{*}=d_{50}\left[\frac{(s-1) g}{v^{2}}\right]^{1 / 3}
$$

(2) Step transport parameter

$$
T=\frac{\left(u_{*}^{\prime}\right)^{2}-\left(u_{*, c}\right)^{2}}{\left(u_{*, c}\right)^{2}}
$$

where, $d_{50}$ particle diameter of $50, s$ specific gravity $(\sigma / \rho), \sigma$ density of the sediment, $\rho$ density of the water, $V$ kinematic viscosity, $u_{*}=\left(\mathrm{g} 0.5 / \mathrm{c}^{\prime}\right) \mathrm{U}$ friction velocity of flow with reference to particle, $U$ average speed of flow, c' coefficient of the particle with Chezy reference.

\subsection{Lagrangian Model}

Models of sediment transport in turbulent flow are determined with another aspect. The model of Van Rijn can be classified as a deterministic model known as Eulerian model. There is another aspect of sediment transport modeling. This probabilistic model known as lagrangian model. The fundamentals of this model were made by using the parameters [13] pick up rate and step length. More sophisticated model was developed by Nakagawa and Tsujimoto [14] that explains about the mechanism step length using a probabilistic function. This model was known as an Eulerian model. The model of Nakagawa and Tsujimoto is very sensitive especially in term of changing the input and favorable in explaining the phenomenon. The mechanisms of the sediment pulling up and transport of saltation and rolling had been 
searched. Gotoh clarified the mechanism of transport based on the model of Nakagawa and Tsujimoto. Lagrangian model was used in this study with following reasons allows the simulation of motion of each particle of the sediment and unifies the phenomenon of sediment transport.

The first reason was used because the existences of coarse sediment near the downstream area. The Lagrangian model has its advantage when the diameter of the material is large. The second reason was it easily explained about transition of the sediment transport phenomenon. When a particle of sediment is pulled up and picked up by runoff, it will be transported by the macro turbulence. The equation of motion of the particle in the flow, according to Newton's second law can be written as

$$
M \overrightarrow{a_{s}}=\overrightarrow{F_{D}}+\overrightarrow{F_{L}}+\overrightarrow{F_{g}}
$$

where, $M$ virtual mass of sediment particle, $\vec{a}_{s}$ sediment particle acceleration, $\vec{F}_{D}$ drag force hydrodynamic, $\vec{F}_{L}$ ascending hydrodynamic force, $\vec{F}_{g}$ force of gravity. Assumed that the particle is a sphere, the quantities above are:

$$
\begin{gathered}
M=\rho\left(\frac{\sigma}{\rho}+C_{M}\right) A_{3} d^{3} \\
\vec{F}_{g}=\rho\left(\frac{\sigma}{\rho}+C_{M}\right) \vec{g} A_{3} d^{3} \\
F_{D}=-\frac{1}{2} C_{D} \rho A_{2} d^{2}\left|\vec{u}_{x}\right| \vec{u}_{x}+\rho\left(1+C_{M}\right) A_{3} d^{3} \frac{\overrightarrow{D u_{f}}}{D t}
\end{gathered}
$$

where, $\vec{u}_{r}=\vec{u}_{p}-\vec{u}_{f}$ relative velocity of the particle. According to the work of Rijn [11], the hydrodynamic forces in turbulent flow and upward can be written as

$$
\vec{F}_{L}=-\alpha_{L} \rho v^{1 / 2} d^{2}\left(\frac{d \vec{u}}{d n}\right)
$$

where, $n$ vector perpendicular to the vector $\vec{u}_{r}, \alpha_{L}$ ancestry coefficientvarying according to the number.

\subsection{Continuity Equation for Water}

$$
\frac{\partial}{\partial t} f_{L} \rho+\frac{\partial}{\partial x_{j}} f_{L} \rho \overline{u_{j}}=0
$$

$$
\begin{gathered}
\frac{D\left(f_{L} \rho \overline{u_{i}}\right)}{D t}=\frac{\partial}{\partial_{j}}\left(1+f_{a}+2.5 f_{S}\right) \mu\left(\frac{\overline{a u}_{i}}{\partial_{j}}+\frac{\overline{\partial u}_{j}}{\partial_{i}}-\frac{2}{3} \frac{\bar{a}_{k}}{\partial \partial_{k}} \delta_{i j}\right) \\
-\frac{\partial \bar{P}}{\partial x_{i}}+f_{L} \rho g_{i}-\nabla\left\{f_{L} \rho\left(R_{i j}+L_{i j}+C_{i j}\right)\right\}+S_{p i}
\end{gathered}
$$

Reynolds term

$$
\begin{gathered}
R_{i j}=-2 K S_{i j}, S_{i j}=\frac{1}{2}\left(\frac{\overline{\partial u_{i}}}{\hat{\alpha}_{j}}+\frac{\overline{\partial u_{j}}}{\hat{\alpha}_{i}}\right), K=\left(C_{S} \Delta\right)^{2}\left[2 S_{i j} S_{i j}\right]^{1 / 2} \\
\Delta^{2}=\left(\Delta x^{2}+\Delta y^{2}+\Delta z^{2}\right) / 3
\end{gathered}
$$

Leonards term

$$
\begin{gathered}
L_{i j}=-\frac{\Delta_{k}^{2} \partial^{2}\left(\overline{u_{i} u_{j}}\right)}{24 \partial_{k} \partial_{k}}, C_{i j}=-\frac{\Delta_{k}^{2} \bar{u}_{i} \partial^{2}\left(\overline{u_{j}}\right)}{24 \alpha_{k} \partial_{k}}-\frac{\Delta_{k}^{2} \bar{u}_{j} \partial^{2}\left(\overline{u_{i}}\right)}{24 \partial_{k} \partial_{k}} \\
S_{p i}=-\frac{\partial \overline{M_{p i}}}{\partial t}, \overline{M_{p i}}=V^{-1}\left(\sum_{k=0}^{k=N S} m_{p k} u_{p i k}\right)
\end{gathered}
$$

in which, $f_{L}, f_{g}, f_{s}$ are volumetric ratio of liquid (water), gas (air) and solid (sediment) phases, respectively, $i, j, k=1,2,3, \quad u_{i}$ velocity component of water in I direction, $\rho$ water density, $p$ water pressure, $g$ gravity force (when $\mathrm{i}=3$ ) , $R_{i j}, C_{i j}, L_{i j}$ are Reynolds, Cross, Leonards terms, respectively, determined by Kano et al. [15] Cs: Smagolinsky [16] coefficient, $\mu$ is water viscosity, $\Delta x, \Delta y$ grid spacing for each direction $V$ Cell volume, $S_{p i}$ is negative production term for flow field by particle movement, $m_{p k}$ specific mass of particle $k$, $u_{p i k}$ : i direction velocity component of particle $k$.

Changes in momentum in solid phases calculated by lagrangian particle model will be transferred into momentum changes in the liquid phase through negative production term determined in Eq. (13). Similarly the loss of the energy when collision occurs also transferred into liquid phase through this negative production term. This effect shown in Fig. 2.

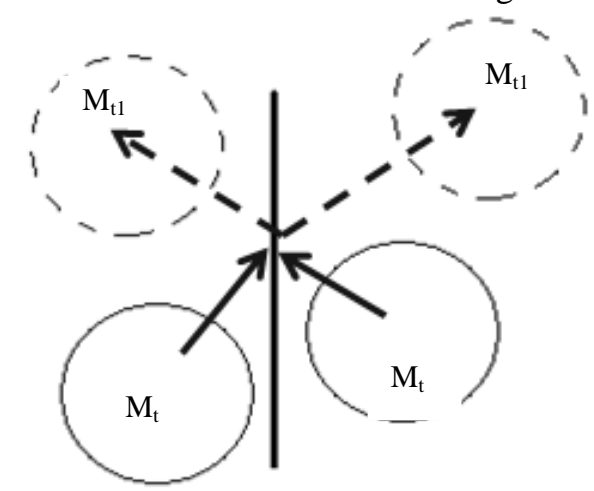

Fig. 2 The effect of negative production term for collision particles. 
Lagrangian sediment transport equation:

$$
\begin{aligned}
& \rho\left(\frac{\sigma}{\rho}+C_{M}\right) A_{3} d^{3} \frac{d \vec{u}_{p}}{d t} \\
& =-\frac{1}{2} C_{D} \rho\left|u_{r}\right| \frac{r}{u_{r}} A_{2} d^{2}+\rho\left(1+C_{M}\right) A_{3} d^{3} \frac{d \underline{u}_{f}}{d t} \\
& +\rho\left(\frac{\sigma}{\rho}-1\right) A_{3} d^{3}\left(g_{i}-\mu_{f} g_{j}\right) \\
& C_{D}=0.4+\frac{24}{R_{e}} ; R_{e}=\frac{\left|u-u_{p}\right| d}{v}
\end{aligned}
$$

in which $C_{D}$ drag coefficient, $\vec{u}_{p}$ velocity vector of sediment particle (for each diameter), $\vec{u}_{f}$ water phase velocity vector $\vec{u}_{r}=\vec{u}_{p}-\vec{u}_{f}, A_{2}=\pi / 4, A_{3}=\pi / 6$, $d$ diameter of the sediment, $C_{M}$ virtual mass coefficient $(=0.2,0.35), s$ density of sediment particle, $\rho$ water density, $\varepsilon$ shadding coefficient determined only when sediment is in the bed and shaded by other sediment, $\mu_{f}$ friction coefficient only works when sediment is at the bottom. This term was determined according to Nakagawa et al. [17] for the simulation of successive saltation movement as followed Gotoh's [6] method. The lagrangian air bubble transport equation is introduced similarly as:

$$
\begin{aligned}
& \rho \beta A_{3} a^{3} \frac{d \vec{u}_{a}}{d t} \\
& =-\frac{1}{2} C_{D a} \rho\left|\vec{u}_{r a}\right| \vec{u}_{r a} A_{2} a^{2}+\rho \beta A_{3} a^{3} \frac{D \vec{u}_{f}}{D t} \\
& -\rho g A_{3} a^{3}
\end{aligned}
$$

where, $\beta$ is virtual mass, given as $0.5, \rho$ is water density, $a$ is an air bubble diameter, $C_{D a}$ friction coefficient of air bubble, which is 2.6. The Adam-Bashforth method was used for time integration.

The air entrainment process was treated from the water surface. The air entrainment was treated by distributing air bubble marker particles in the sub-cells of the surface cells with no water marker cell. These distributed air bubble marker particles are transported according to the surface air drag coefficient which is assumed to be 1.0. The air entrainment by the air bubble capitation is simulated by distributing the air bubble marker particles in the inner air cells. The air bubble pairing and dividing cannot be simulated in this study.

\section{Results and Discussions}

This section the authors only present results and discussions for the $25^{\circ}$ slope angle case study. Tracing positions of $10 \mathrm{~mm}$ and $2.5 \mathrm{~mm}$ particles are represented by black and white colors, respectively. The first case is initial, no liquid-phase flow case. Fig. 3a shows the captured image for this case. Six different shapes were employed to represent the particle path line. In this case no small particle images were captured. This means, big particles move faster rather than small particles. The longest distance of movement for big particles (circle shape) is $14.09 \mathrm{~cm}$ and the shortest (diamond shape) is $5.74 \mathrm{~cm}$. The average velocity for this case is $0.19 \mathrm{~cm} / \mathrm{ms}$. Suwa [18] claim that the cause of the convergence of large particles at the front of a debris flow is caused by their faster longitudinal velocities than the surrounding small particles, a vertical particle-segregation concept is adopted here to explain the convergence of large particles at the front. Takahashi [19] verified this theory in previous flume experiments study. The velocity varies from zero at the bottom to a high value in upper layer, and the mean velocity is somewhere in between the bottom and surface values, if grain sorting arises in the following layer and coarser particles are transferred to the upper part, those particle would be transported faster than the mean propagating velocity of the debris flow front.

Small particle images can be seen starting with liquid-phase flow. A group of white lines concentrated near the bottom while the black lines are at the upper part. It shows that big particles move upward and in contrast for small particles. The average velocities of big particles are faster than the small ones. The velocity is $2 \mathrm{~cm} / \mathrm{ms}$ faster. Fig. 3b shows particle tracing captured by HSVC for starting with liquid-phase flow. Same phenomenon happens for intermediate (2 s) case. Big particles move upward and faster than small particles. The average velocity of the big particles is 


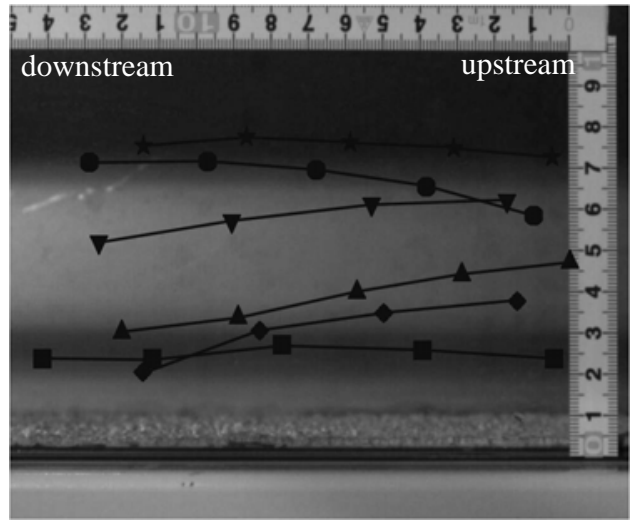

(a)

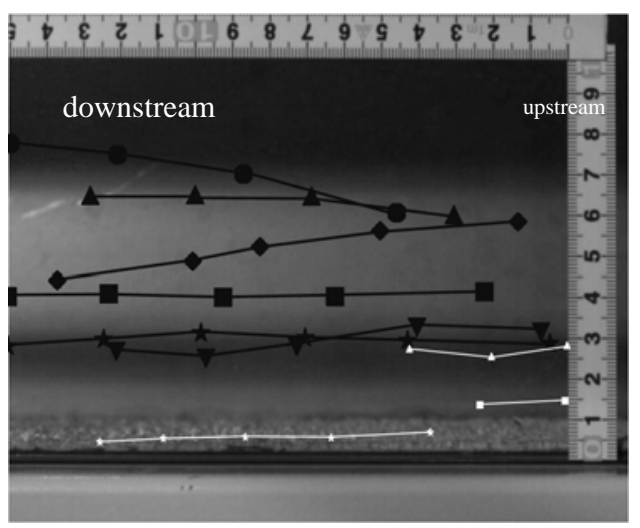

(b)

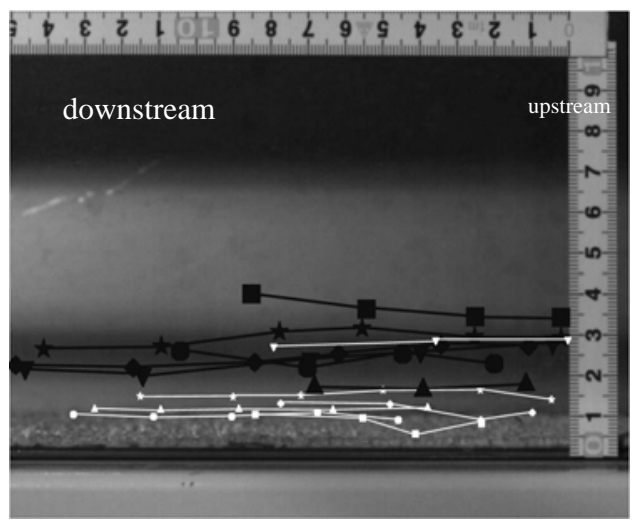

(c)

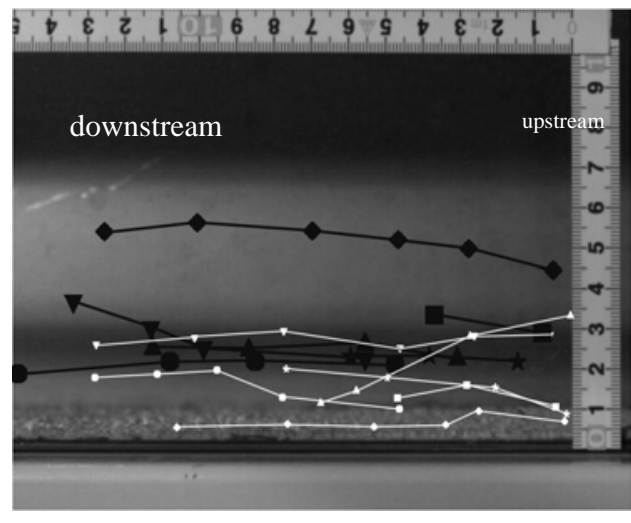

(d)

Fig. 3 Particle tracing captured by HSVC for $25^{\circ}$ (a) initial, no liquid-phase flow (b) starting with liquid-phase flow (c) intermediate, $2 \mathrm{~s}$ (d) last, $4 \mathrm{~s}$.
$4 \mathrm{~cm} / \mathrm{ms}$ faster than the small particles. From this captured image, we can understand the characteristics of particle movements between particles of different sizes [20].

The particle routing segregation image can be referred in Fig. 4. The footage images duration is from $1.0 \mathrm{~s}$ to $4.0 \mathrm{~s}$. Each image was captured at every $0.5 \mathrm{~s}$. The experimental and numerical velocities shown a wider range of value from the smallest of $0.156 \mathrm{~cm} / \mathrm{ms}$ to the highest $0.249 \mathrm{~cm} / \mathrm{ms}$. The numerical mean velocity result is slightly lower than the experimental mean value. The values of each case are $0.192 \mathrm{~cm} / \mathrm{ms}$ and $0.212 \mathrm{~cm} / \mathrm{ms}$ respectively. The numerical results predicted about $10.4 \%$ lower in mean velocity than experimental result.

$1.0 \mathrm{~s}$

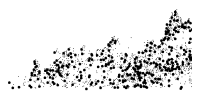

$1.5 \mathrm{~s}$

$2.0 \mathrm{~s}$

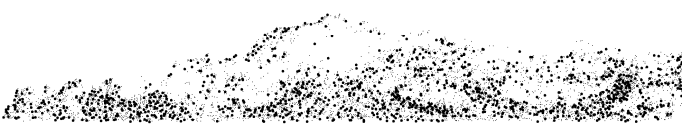

$2.5 \mathrm{~s}$

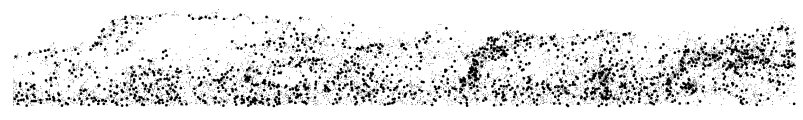

$3.0 \mathrm{~s}$

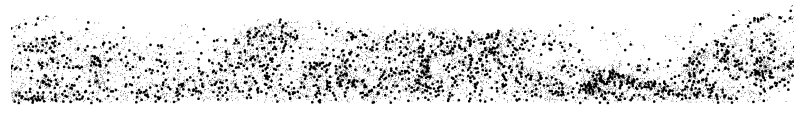

$3.5 \mathrm{~s}$

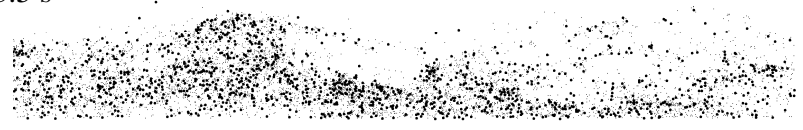

$4.0 \mathrm{~s}$

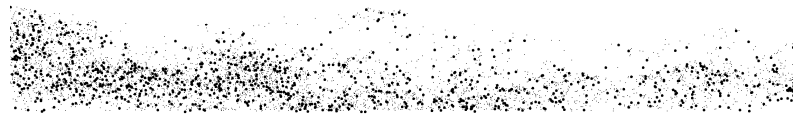

Fig. 4 The HD2DM result of the particle distributions near downstream, $\mathrm{C}_{\mathrm{m}}=\mathbf{0 . 3 5}$. 
In intermediate case (big particle) for experimental and numerical, mean velocity for experimental is recorded as $0.194 \mathrm{~cm} / \mathrm{ms}$ which is $0.01 \mathrm{~cm} / \mathrm{ms}$ slower compared to the numerical result. It means that, the numerical result predicted about $4.9 \%$ higher in mean velocity than experimental result. The different of mean velocity of numerical and experimental value is 5.5\% lower compared to the initial case (big particle).

The highest percentage of numerical model prediction compared with experimental mean velocity result can be seen in last case (small particle). The mean average value is $22.61 \%$. The highest velocities in experimental and numerical are $0.186 \mathrm{~cm} / \mathrm{ms}$ and $0.253 \mathrm{~cm} / \mathrm{ms}$ while the lowest velocities are 0.113 $\mathrm{cm} / \mathrm{ms}$ and $0.156 \mathrm{~cm} / \mathrm{ms}$ respectively. The numerical model prediction compared with experimental mean velocity in intermediate case (small particle) is $17.19 \%$.

The particles routing distribution produced by the HD2DM for two different virtual mass coefficients ( 0.2 and 0.35) cannot be concluded just referred to the particles movement mechanism. Therefore, the mean average velocity of numerical compared with experimental model had to be referred. The comparison of particles mean velocity distribution can be seen in Fig. 5. Bigger particles flow at the upper part, while smaller particles attach near to the bottom. The bigger particle movements were concentrated at the upper (high velocity) part of the fluid, while small particles were concentrated near the bottom (low velocity). This phenomenon happen as the same as observed in the experimental study. However still some modifications have to be made.

We can conclude that the virtual mass $\left(\mathrm{C}_{\mathrm{M}}\right)$ value is most important criteria should be considered. This because the virtual mass values will affect ascending hydrodynamic force and gravity force. For instance, when the virtual mass is low (0.2) the ascending hydrodynamic and gravity force are 0.42 and 0.58 respectively.
But when the virtual mass is high (0.35) the ascending and gravity force become 0.45 and 0.55 respectively. Here we can say that, when the virtual mass is high it means the ascending hydrodynamic force will become high, but the gravity effect becomes low. In case of virtual mass become low, the ascending hydrodynamic force also become low but the gravity force will become high. Eqs. (17) and (18) below shows the sediment particle acceleration due to ascending hydrodynamic and gravity forces. These two equations were derived from Eqs. (3) and (14).

$$
\begin{gathered}
\frac{d u_{p}}{d t}=a_{s}=\frac{F_{g}}{M}=\frac{\rho\left(\frac{\sigma}{\rho}-1\right)}{\rho\left(\frac{\sigma}{\rho}+C_{m}\right)} \\
\frac{d u_{p}}{d t}=a_{s}=\frac{F_{L}}{M}=\frac{\rho\left(1+C_{m}\right)}{\rho\left(\frac{\sigma}{\rho}+C_{m}\right)}
\end{gathered}
$$

In previous study, conducted by Gotoh (1992) and Tchen (1947), the value of virtual mass coefficient (0.5) was fitted in their model. In this study, after the calibration processes had been done, the best fitted virtual mass coefficient value was 0.35 . The mean average velocity values show ranging from $3.1 \%$ to $13.5 \%$ is obtained from the velocity results of numerical compared to the experimental near the upstream is $1.2 \%$ to $22.61 \%$ near the downstream.

For further understanding, Table 1 shows the mean average velocity of numerical model compared with experimental near the downstream. In this study, the effects of interaction between sediment particles are considered only for single collision, in other term, multiple collision effects are not considered in this study. We verified the applicability of this assumption through HSVC study by checking whether multiple collisions occurs in this concentration, and confirmed that consideration of only single collision effect is enough for this study. In the future, we suggest that study about the effects of interaction between sediment particles with multiple collision effects should be done to observe the particle segregation movements. 
Table 1 The mean average velocity of numerical compared to experimental.

\begin{tabular}{llllll}
\hline \multirow{2}{*}{ Virtual mass, Cm } & Initial & \multicolumn{2}{c}{ Intermediate (2 s) } & \multicolumn{2}{c}{ Last (4 s) } \\
\cline { 2 - 6 } & Big particle (\%) & Big particle (\%) & Small particle (\%) & Big particle (\%) & Small particle (\%) \\
\hline 0.2 & 48.3 & 34.7 & 26.2 & 33.1 & 16.7 \\
0.35 & 10.4 & 4.9 & 17.19 & 1.2 & 22.61 \\
\hline
\end{tabular}

Initial case
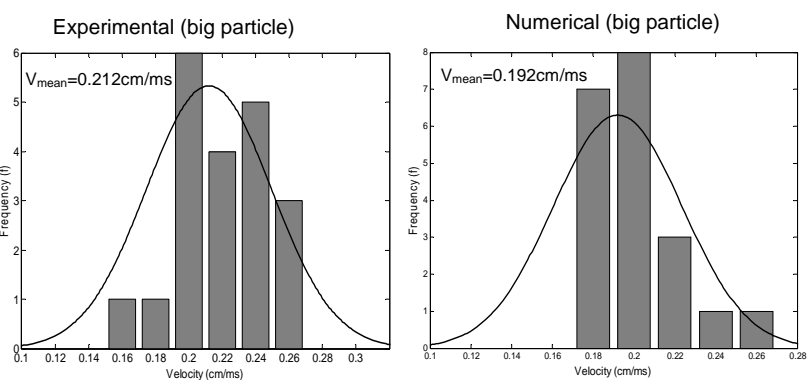

Last (4s) case
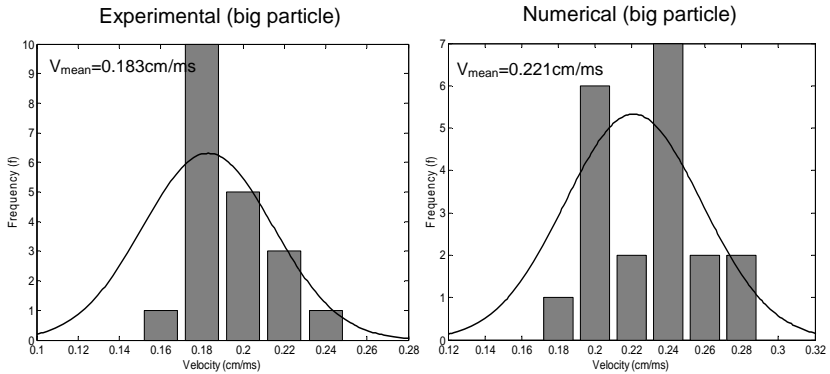

Last (4s) case
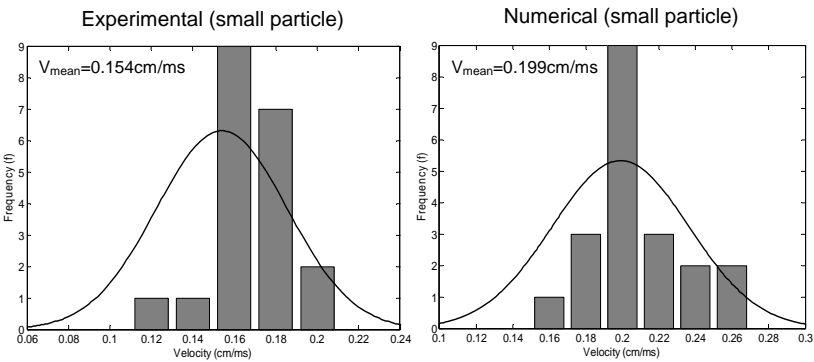

Fig. 5 Comparison of experimental and numerical particles velocity frequency distribution at different cases near the downstream (virtual mass value, 0.35 ).

\section{Conclusions}

Overall from this study shows, the numerical results are in good agreement with the experimental result. It can be concluded that the calibration processes for velocity were successful. The value of virtual mass was found as one of the most important criteria that should be considered in calibration process, as this parameter dominates fundamental characteristics of sediment particle movement in the lagrangian numerical scheme.

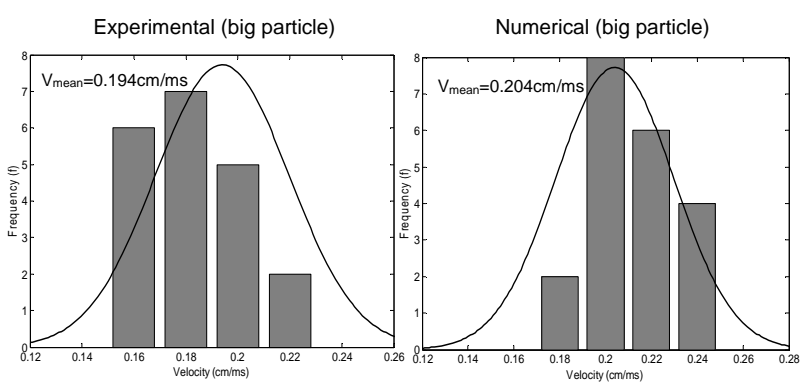

Intermediate (2s) case

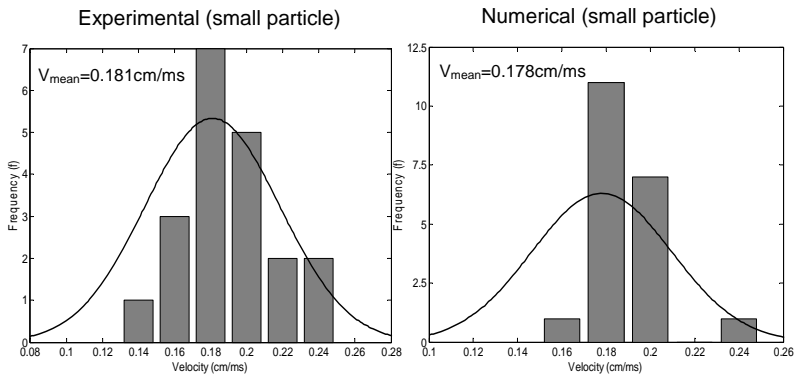

$$
\text { . }
$$


show ranging from $1.2 \%$ to $22.61 \%$ is obtained from the velocity results of numerical compared to the experimental. Furthermore, the particles routing distribution produced by the HD2DM for two different virtual mass coefficients ( 0.2 and 0.35 ) cannot be concluded just referred to the particles routing distributions. Therefore, the mean average velocity of numerical compared with experimental model had to be referred.

\section{Acknowledgment}

This research was carried out with financial support from the Japanese Science and Technology Agency (JST), Japan International Cooperation Agencies (JICA), Science and Technology Research Partnership for Sustainable Development (SATREPS) and also GCOE-HSE, Kyoto University. Also thanks to Mr. Fujiki Shigeo and Ms. Maja Ostric for their assistance in performing the experiments.

\section{References}

[1] J. M. Martinez, G. Avila, A. Agudelo, R. L. Schuster, T. J. Casadevall and K. M. Scott, Landslides and debris flows triggered by the 6 June 1994 Paez earthquake, Southwestern Colombia, Landslide News 9 (1995) 13-15.

[2] V. Villi and A. Dal Pra, Debris flow in the upper Isarco valley, Italy-14 August 1998, Bulletin of Engineering Geology and the Environment 61 (1) (2002) 49-57.

[3] O. Hungr, G. C. Morgan and R. Kellerhals, Quantitative analysis of debris torrent hazards for design of remedial measures, Canadian Geotechnical Journal 21 (4) (1984) 663-677.

[4] D. F. VanDine, Debris flows control structures for forest engineering, in: Working Paper, Britsh Columbia Ministry of Forest Research Program, Victoria, 1996.

[5] D. O. K. Lo, Review of natural terrain landslide debris-resisting barrier design: GEO Report, Geotechnical Engineering Office, Civil Engineering Department, The Government of Hong Kong Special Administrative Region, 2000.

[6] H. Gotoh, Study of sediment paricle dynamics and its application for movable bed, PhD Dissertation, Kyoto University, 1992.
[7] C. T. Crowe, M. P. Sharma and D. E. Stock, The particle source in cell (PSI-CELL) model for gas droplet flows, Journal of Fluid Engineering 99 (2) (1977) 325-332.

[8] C. M. Tchen, Equation of the motion for a particle suspended in a homogenous field, PhD Dissertation, Delft University, 1947.

[9] Y. Takemoto, A computer code for time-dependent, viscous, incompressible fluid flows using the third-order upwind finite-difference scheme called "QUICK 132 y0/QMAC2D”, Journal of Super Computer 17 (6) (1983).

[10] T. Sakai, T. Mizutani, H. Tanaka and Y. Tada, Numerical simulation of breaking wave on slope, in: Bulletin of 34 Coastal Engineering Symposium, JSCE (Japan Society of Civil Engineers) (1987) 71-75.

[11] V. Rijn, C. Leo, Sediment transport, part 1: Bed load transport, Journal of Hydraulic Engineering, ASCE 110 (10) (1984) 1431-1456.

[12] V. Rijn, C. Leo, Sediment transport, Part 2: Suspended load transport, Journal of Hydraulic Engineering, ASCE 110 (11) (1984) 1613-1641.

[13] A. Einstein, The bed load function for sediment transportation in open channel flows, Technical Bulletin, USDA (U.S. Dept. of Agriculture), Soil Conservation Service, 1950, p. 78.

[14] H. Nakagawa, T. Tsujimoto, Sand bed instability due to bed load motion, Journal of Hydraulic Engineering 106 (12) (1980) 2029-2051.

[15] M. Kano, T. Kobayashi, T. Ishihara, Prediction of turbulent flow in two-dimensional channel with turbulent promoters, Mechanical Engineering Research 28 (50) (1985) 2940-2947.

[16] J. Smagolinsky, Monthly Weather Revision 93 (1963) 99.

[17] H. Nakagawa, T. Tsujimoto, T. Hosokawa, Stochastic Sediment Movement in Movable Bed, DPRI Annual Bulletin, Kyoto University, 1978.

[18] H. Suwa, Focusing mechanism of large boulders to a debris flow front trans., Japanese Geomorphological Union, Kyoto, Japan 9 (3) (1988) 151-178.

[19] T. Takahashi, Debris flow on prismatic open channel, Journal Hidraulic Division 106 (3) (1980) 381-396

[20] M. A. Z. Remy Rozainy, Y. Yamashiki, K. Takara, T. Matsumoto and T. Takahashi, Experimental study of debris routing particle segregation with constant discharge, in: 13th International Summer Symposium of the International Activities Committee, Japan Society of Civil Engineering, Uji, Kyoto University, Japan, 2011, pp. 231-234. 